\title{
Un enfoque cognitivo del análisis del discurso: el caso de informes de Derechos Humanos en Chile
}

A cognitive approach to discourse analysis: the case of Human Rights reports in Chile

\author{
Barbara De Cock ${ }^{1}$ \\ Université Catholique de Louvain \\ Daniel Michaud Maturana ${ }^{2}$ \\ Université Catholique de Louvain \\ Marcela Alejandra Ruiz Zúñiga ${ }^{3}$ \\ Universidad Alberto Hurtado
}

\begin{abstract}
RESUMEN: Mediante el análisis discursivo de informes sobre los Derechos Humanos en Chile producidos en diferentes momentos históricos, mostramos cómo los hallazgos de la lingüística cognitiva pueden contribuir a la comprensión de fenómenos sociales. Concretamente, analizamos tres aspectos discursivos: (i) la gradualidad de la identificación de víctimas y perpetradores, (ii) el grado de intencionalidad que se atribuye a la acción y las representaciones más o menos prototípicas del agente, y (iii) la descripción de espacios que activa la noción de ciertos perpetradores. Los resultados muestran que los informes de la OEA, escritos durante la dictadura, ofrecen información más detallada sobre los responsables de los abusos de Derechos Humanos que el Informe Rettig, escrito al inicio de la transición a la democracia. Asimismo, este estudio muestra cómo la lingüística cognitiva puede contribuir al análisis de discursos de la (no) atribución de la responsabilidad.
\end{abstract}

Palabras-clave: Lingüística cognitiva; Análisis del discurso; Derechos Humanos; Chile; Agentividad.

RESUMO: Mediante a análise discursiva de relatórios sobre Direitos Humanos no Chile produzidos em diferentes momentos históricos, mostramos como as propostas da linguística cognitiva podem contribuir para a compreensão de fenômenos sociais. Concretamente, analisamos três aspectos discursivos: (i) a gradualidade da identificação de vítimas e torturadores; (ii) intencionalidade que se atribui a ação e as representações mais ou menos prototípicas do agente; e (iii) a descrição de espaços que ativa a noção de certos torturadores. Os resultados mostram que os relatórios da OEA, escritos durante a ditadura, oferecem informação mais detalhada sobre os responsáveis dos abusos de Direitos Humanos que o Informe Rettig, escrito no início da transição para a democracia. Além disso, este estudo mostra como a linguística cognitiva pode contribuir para a análise de discurso da (não) atribuição da responsabilidade.

\footnotetext{
${ }^{1}$ Faculté de Philosophie, Arts et Lettres, Institut Langage et Communication, Pôle de Recherche en Linguistique, Prof. Dr. (associate professor).

${ }^{2}$ Faculté de Philosophie, Arts et Lettres, Institut Langage et Communication, Pôle de Recherche en Linguistique, Dr. Collaborateur scientifique / Zuyd University of Applied Sciences, staff member.

${ }^{3}$ Facultad de Filosofía y Humanidades, Departamento de Lengua y Literatura, Doctor en Lingüística.
} 
Palavras-chave: Linguística cognitiva; Análise do discurso; Direitos Humanos; Chile; Agentividade.

ABSTRACT: Through a discursive analysis of reports on human rights in Chile from different moments in recent history, we show how finding of cognitive linguistics can contribute to a better understanding of social phenomena. More concretely, we analyze three discursive aspects: (i) the graduality of the identification of victims and perpetrators, (ii) the degree of intentionality that is attributed to the actions and the (more or less) prototypical representations of the agent, and (iii) the description of spaces that activate the notion of certain perpetrators. The results show that the OEA reports, written during the dictatorship, offer more detailed information about those responsible for human rights violations than the Rettig report, written at the beginning of the transition towards democracy. Moreover, this study shows how cognitive linguistics can contribute to the analysis of discourses concerning the (non-)attribution of responsibility.

Keywords: Cognitive linguistics; Discourse analysis; Human rights; Chile; Agentivity. 


\section{Introducción}

Este artículo muestra cómo la lingüística cognitiva puede contribuir al análisis del discurso mediante el estudio de informes sobre los Derechos Humanos en Chile y, más concretamente, mediante un análisis que contrasta un informe publicado bajo la dictadura con un informe publicado durante el periodo transicional hacia la recuperación de la democracia. Mostramos, pues, que un análisis del discurso inspirado en la lingüística cognitiva permite describir con mayor claridad las diferencias existentes entre dos informes publicados en momentos claves.

Los objetivos son pues tanto generales como específicos. A nivel general teórico, mostraremos cómo un enfoque cognitivo puede contribuir a comprender las conceptualizaciones representadas en un discurso, con atención particular al concepto de punto de vista. A nivel específico, queremos mostrar los contrastes en los informes con respecto a: (i) el grado de especificidad con que se describe a los perpetradores y a las víctimas, (ii) la intencionalidad de los actos de los perpetradores y (iii) el uso del espacio para evocar un marco y sus participantes. Tras una presentación de la lingüística cognitiva (2) y del contexto histórico de los informes analizados (3), procederemos entonces al análisis de los informes (4) y a la presentación de las conclusiones (5).

\section{La lingüística cognitiva}

La lingüística cognitiva considera que en la producción lingüística creamos conceptualizaciones de la realidad y que, en consecuencia, formas o construcciones lingüísticas distintas representan conceptualizaciones diferentes. Si bien en la lingüística cognitiva se han realizado muchos trabajos sobre aspectos léxicos y sintácticos, también se han realizado varios análisis del discurso desde una perspectiva cognitiva (HART; LUKE ̌́, 2009; KNAPTON; RUNDBLAD, 2018), incluyendo asimismo aspectos multimodales (SAMBRE, 2017, SAMBRE; FEYAERTS, 2017). Efectivamente, tomando en cuenta la idea central de la lingüística cognitiva sobre el lenguaje como herramienta de conceptualización de la realidad, el análisis propuesto en esta contribución no se centrará en un fenómeno o nivel lingüístico concreto, sino que muestra la interacción entre varios fenómenos concretos a fin de arrojar luz sobre cómo se representa a los responsables de abusos de Derechos Humanos en 
Barbara De Cock; Daniel Michaud Maturana. Marcela Alejandra Ruiz Zuñiga. Un enfoque cognitivo del análisis del discurso: el caso de informes de Derechos Humanos en Chile.

dos informes comparados. Con tal objetivo nos inspiraremos en los siguientes conceptos de la lingüística cognitiva.

En primer lugar, en los focos de atención (TALMY, 2000). En la lingüística cognitiva, se considera que el lenguaje y las alternancias entre distintas construcciones corresponden a conceptualizaciones en que la atención se centra en aspectos diferentes. Así, construcciones alternativas pueden referirse a un mismo acontecimiento, pero de forma distinta como consecuencia del foco escogido.

En segundo lugar, nos inspiramos en la teoría de marcos (FILLMORE, 2003). Esta teoría permite analizar cómo ciertas expresiones activan otros conceptos relacionados. En ese sentido, la teoría de marcos establece la relación entre las relaciones semánticas y el conocimiento del mundo. Así, un verbo como enseñar activa de modo más prominente las nociones de profesora, alumno que la de fontanero, por ejemplo. Veremos en el marco de nuestro estudio que la selección de cierto verbo o sustantivo determina el marco y los participantes que se activan.

Por último, nos basaremos también en los grados de variabilidad de la prototipicidad del agente según la preposición que introduce la frase nominal agente y las características semánticas de la misma (véase DELBECQUE, 2014). Esta variabilidad muestra que otras estructuras que la de complemento de agente prototípico - que consiste en por + sustantivo con referente humano - pueden funcionar como agente o, por lo menos, evocarlo. Este enfoque nos permitirá mirar más allá del agente prototípico humano introducido por la preposición por para tomar en cuenta el abanico de estrategias posibles para evocar el agente.

\section{Contexto histórico de los informes}

Compararemos en este análisis el informe de la Organización de Estados Americanos (OEA) de 1974, al inicio de la dictadura, con el Informe Rettig de 1991, al inicio del periodo de transición. Utilizaremos en este estudio la paginación de la re-edición de 1996 públicamente disponible en línea. A continuación, ofrecemos mayor información sobre el contexto de redacción de dichos informes.

La defensa de los Derechos Humanos se difunde habitualmente a través de informes que pretenden que diversos actores sociales (estados, comunidad nacional e internacional, organismos internacionales) conozcan los hechos denunciados y tomen medidas para proteger a los sujetos vulnerados (RUIZ; BERNASCONI, 2019). Entre los informes de Derechos 
Humanos se encuentran los producidos por organizaciones no gubernamentales, organismos internacionales y Comisiones de Verdad y Reconciliación en sociedades postconflicto, como sucedió en Argentina, Guatemala, Sudáfrica, Timor-Leste, África del Sur, que han enfrentado graves violaciones a los Derechos Humanos y procesos de justicia transicional (FERRARA, 2017; GUTHREY, 2015; WILSON, 2001; ANTHONISSEN; BLOMMAERT, 2006; BRANTS; KLEP, 2013; HAYNER, 2008).

La sociedad chilena también enfrentó graves violaciones a los Derechos Humanos entre 1973-1990. La dictadura militar encabezada por Pinochet practicó la represión sistemática a través de la desaparición forzada, la ejecución, la tortura, la prisión, el exilio, los despidos. La Doctrina de Seguridad Nacional promovía la guerra antisubversiva y la creación del concepto de "enemigo interno", con el fin de eliminar a las organizaciones que habían apoyado los cambios sociales durante el gobierno de la Unidad Popular (1970- 1973). La represión militar contó con organismos de seguridad y servicios de inteligencia (Dirección de Inteligencia Nacional - DINA, Centro Nacional de Informaciones - CNI), con el control de los medios de comunicación, con el apoyo de sectores de la sociedad civil y con la inacción del poder judicial (BRUNNER, 1981; VALDIVIA ORTIZ DE ZÁRATE, 2003; 2010; DORAT GUERRA; WEIBEL BARAHONA, 2012).

Sin embargo, apenas se produjo el golpe de Estado, hubo organizaciones religiosas y civiles que se opusieron. El 6 de octubre de 1973, fue creado el Comité de Cooperación para la Paz en Chile (COPACHI) con la concurrencia de diversas confesiones religiosas: Iglesia Católica, Iglesia Ortodoxa, Iglesias Evangélicas y Comunidad Israelita. Entre los principales objetivos del COPACHI estaba proporcionar asistencia material y legal a los familiares y víctimas de violencia política, y también recolectar y registrar denuncias de hechos que lesionaban la dignidad humana. No obstante, este organismo no pudo resistir la presión del gobierno militar que impuso su disolución en 1975. Este hecho marca la transición del COPACHI a otro organismo que se crea bajo el amparo de la Iglesia Católica: la Vicaría de la Solidaridad (1976-1992).

En el inicio de la dictadura cívico-militar, las primeras denuncias sobre la situación chilena son conocidas internacionalmente a través de los primeros informes de organismos no gubernamentales elaborados por Amnistía Internacional y la Comisión Internacional de Juristas tras visitar el país (AMNESTY INTERNATIONAL, 1974; MAC DERMOT; MADLENER; OLIVER, 1974). Durante las visitas, las comisiones de ambas organizaciones se entrevistan con diferentes actores sociales gubernamentales y no gubernamentales. Además de escuchar el testimonio de las víctimas y sus familiares, se reunieron con el Comité de 
Cooperación para la paz en Chile que defendía los derechos de los presos políticos y de los exiliados. De igual modo, la Comisión de Derechos Humanos de la Organización de los Estados Americanos (OEA) publica el primer informe sobre la visita a Chile hecha a fines de julio de 1974 y estipula explícitamente que este es elaborado a raíz de denuncias y quejas sobre los atropellos de los Derechos Humanos. En este informe (CIDH, 1974) se señala que hubo una entrevista con el Cardenal Silva Henríquez y que se tomó conocimiento sobre la presentación masiva de recursos de amparo hecha por el COPACHI. Se señalan como otras fuentes las entrevistas a víctimas, los testimonios de miembros de la comisión OEA que son testigos de operaciones de carabineros y testimonios de abogados. Este informe fue pues elaborado y publicado en plena dictadura y será comparado con el Informe Rettig publicado al inicio del periodo de transición.

Efectivamente, tras el fin de la dictadura y en un nuevo escenario internacional, emergen los conceptos de verdad y justicia como fundamentos para la paz social y como principios de la lucha ante el olvido y la impunidad. Las nuevas autoridades del gobierno democrático de Patricio Aylwin comparten la creencia de que el conocimiento público de la verdad sobre la vulneración de los derechos fundamentales contribuiría al restablecimiento del orden social y permitiría la recuperación de la perdida unidad de la nación. Uno de los mecanismo elegidos fue la constitución de una comisión cuyo fin fue documentar la verdad de los hechos (FERRARA, 2017; LOVEMAN; LIRA, 2000; 2001). La Comisión de Verdad y Reconciliación emplea la documentación proveniente de organismos como el Comité de Cooperación para la paz en Chile y la Vicaría de la Solidaridad que culmina con la publicación del Informe de Verdad y Reconciliación (BERNASCONI; RUIZ; LIRA, 2018), comúnmente llamado "Informe Rettig" por el nombre de su presidente. Los archivos de Derechos Humanos tuvieron un rol relevante no solo para el trabajo de la Comisión, sino también por su documentación que sigue siendo empleada para reconocer y reparar a la víctimas en los procesos judiciales sobre violaciones a los Derechos Humanos (ACCATINO; COLLINS, 2016; GROPPO, 2016). Además de las fuentes expuestas, el Informe Rettig se basa también en informaciones por parte de familiares de víctimas, del Instituto Médico Legal, de la prensa y de fuentes anónimas - posiblemente perpetradores. Sin embargo, el informe no siempre adopta todas las perspectivas posibles y deja fuera de la representación de los hechos la perspectiva de los perpetradores (véase DE COCK; MICHAUD MATURANA, 2018).

Durante el periodo de redacción del Informe Rettig, la comisión trabaja en un contexto de transición en que las Fuerzas Armadas siguen ocupando una posición importante de poder 
en la sociedad. Así, varios miembros de la comisión son próximos a las Fuerzas Armadas y al entorno de Pinochet. Un estudio de la introducción del informe muestra que se presenta a todos los chilenos como responsables (OTEÍZA, 2009; 2010). Las reacciones públicas tras la publicación del informe son por ende divergentes (JOHANSSON MÁRQUEZ, 2001). La posición particular de la comisión se aprecia también en el hecho de que el decreto que establece el marco del informe estipula explícitamente que este no podrá tener consecuencias jurídicas. Grandin (2005, p. 55) resume la particularidad de esta comisión en un periodo de transición de la forma siguiente:

Chile $[\ldots]$ retained the notion that official inquiries into past abuses were needed to reinforce social solidarity but jettisoned, due to political exigencies, the required mechanism that $[\ldots]$ bestowed those inquiries with legitimacy: the ability to hold the worst abusers legally accountable (GRANDIN, 2005, p. 55).

Nuestro análisis se centra en el primer volumen del tomo I, sobre las violaciones a los Derechos Humanos cometidas de septiembre a diciembre 1973. El resto del informe trata el periodo de 1974-1990 cuando el sistema represivo - gestionado sobretodo por la DINA estaba plenamente consolidado (cf. INFORME RETTIG, v. II, p. 449).

Últimamente se añade la discusión sobre el derecho a la memoria (CABEZAS DEL FIERRO et al., 2013) a las consideraciones sobre informes de Derechos Humanos. Este derecho se deriva del derecho a la dignidad, pero no se inscribe tal cual en la legislación chilena (CABEZAS DEL FIERRO et al., 2013, p. 158). Si bien estos informes contribuyen a la construcción de la Memoria Colectiva (HAU, 2005), mostraremos que hay lagunas que van en contra de este derecho.

\section{Análisis}

\subsection{Especificidad}

En primer lugar, compararemos los grados de especificidad con los cuales se presenta a los perpetradores. Si bien en algunos casos se desconocía (y todavía se desconoce) la identidad de los perpetradores de violaciones de Derechos Humanos, en otros casos su identidad ya era conocida. La forma en que se describe y enfoca a los perpetradores implica desde luego cierta conceptualización de la situación. 
Barbara De Cock; Daniel Michaud Maturana. Marcela Alejandra Ruiz Zuñiga. Un enfoque cognitivo del análisis del discurso: el caso de informes de Derechos Humanos en Chile.

La primera constatación crucial es que solo el informe de la OEA contiene nombres concretos de perpetradores, aunque pueden ser aproximativos como en (1). El Informe Rettig, en cambio, no contiene nombres concretos de perpetradores, lo cual se entiende por el marco jurídico en el cual fue realizado (véase DE COCK; MICHAUD MATURANA, 2014; 2018). Siendo realizado después de la dictadura y disponiendo de las informaciones del informe de la OEA y de muchos otros materiales, es evidente que la ausencia de nombres en el Informe Rettig fue el resultado de una decisión y no de una falta de información (por lo menos no para ciertos casos descritos).

(1) No. 2 Detenida en septiembre de 1973 y llevada a la Calle Londres $N^{o} 38$ (no presenta cicatrices, pero acusa como autor de alegadas torturas a los policías llamados aproximadamente Luicajón, Sapalo y Raúl Romo). (OEA)

Ambos informes contienen amplias referencias a grupos de perpetradores identificados por su función oficial, como carabineros en (2) o agentes del estado en (3). Cabe destacar que el informe de la OEA a veces presenta a este grupo con un grado mayor de especificidad, detallando por ejemplo el número de personas como en (2). Además de estos grupos definidos por su función oficial, en ambos informes hay referencias a grupos con aun un menor grado de especificidad, como el uso de individuos en (4) y (5) vs. nombres de función como carabineros. Tales descripciones más vagas pueden deberse tanto a una falta de información como al hecho de que los abusos fueron a veces realizados por personas vestidas de civil que no se identificaban como miembros de las Fuerzas Armadas o como agentes del Estado.

(2) En mi caso, mi detención fue por allanamiento por 20 carabineros, los que apuntaron a mi hija de cuatro años. (OEA)

(3) La Comisión ha llegado a la convicción que Arnoldo Camú Veloso fue ejecutado al margen de todo proceso legal por agentes del Estado. (Rettig 161)

(4) Todo tipo de agresiones de orden sexual, incluida la comisión de actos tan aberrantes como la violación sucesiva por varios individuos, etc. (OEA)

(5) Alrededor de la 00:10 horas del día 18 de septiembre de 1973, todos fueron sacados de los calabozos hacia la calle, donde se encontraron con una fila de individuos vestidos de civil, de negro, con máscaras de vampiro cubriéndoles los rostros. (Rettig 401)

El informe de la OEA añade a estas descripciones también referencias metonímicas de tipo pars pro toto a los perpetradores, como en (6) en donde la expresión Las manos abiertas activa la imagen física del perpetrador. Además, la combinación con una descripción de la acción (golpes simultáneos) y una construcción final (para lesionar el tímpano) intensifica la intencionalidad (véase también la sección siguiente) del perpetrador. 
(6) Golpes simultáneos con las manos abiertas sobre ambos oídos, para lesionar el tímpano. (OEA)

Por último, ambos informes contienen descripciones sin ninguna referencia al perpetrador, tanto en el caso de detenciones $(7,9)$ como de fusilamientos $(8,10)$. Veremos sin embargo en las secciones siguientes que el perpetrador puede ser evocado en el resto de la frase mediante la construcción verbal u otros elementos sintácticos.

(7) Que Luis Vélez, (boliviano), fue detenido y ha desaparecido. (OEA)

(8) Que Sócrates Ponce Pacheco, (ecuatoriano), con 10 años de residencia en Chile, fue fusilado el día 11 de septiembre; vivía en la Torre San Borja, apartamento 141. (OEA)

(9) El 12 de septiembre de 1973, fue detenido Tulio Roberto QUINTILIANO CARDOZO, brasileño, 29 años, ingeniero, militante del Partido Comunista. (Rettig 125)

(10) El 14 de septiembre de 1973 fue fusilado José Eusebio RODRIGUEZ HERNANDEZ de 24 años, obrero, militante del Movimiento de Izquierda Revolucionaria (MIR). (Rettig 134)

Resumiendo, en este primer acercamiento al tema, constatamos que el informe de la OEA redactado durante la dictadura contiene referencias más específicas e incluso llega a identificar a los perpetradores en algunas ocasiones. El Informe Rettig, en cambio, no tiene referencias más específicas que la descripción de un grupo de un número indefinido de personas descrito por su función oficial, lo cual repercute en su valor informativo (WINN, 2007).

\subsection{Intencionalidad}

En esta sección, nos detendremos en las distintas construcciones utilizadas para expresar la vulneración de Derechos Humanos, con atención particular a la expresión de la intencionalidad. Si bien encontramos en ambos informes grados variables de intencionalidad, mostraremos que los grados menores están ausentes en el informe de la OEA. Ambos informes contienen verbos activos cuyo significado implica una acción intencionada como en (11) y (12). Ambos informes emplean también construcciones pasivas en que la intencionalidad se expresa por el contenido del verbo y la mención del agente humano como en (13) y (14) (por un Capitán y por personal del ejército).

(11) En mi caso, mi detención fue por allanamiento por 20 carabineros, los que apuntaron a mi hija. (OEA) 
Barbara De Cock; Daniel Michaud Maturana. Marcela Alejandra Ruiz Zuñiga. Un enfoque cognitivo del análisis del discurso: el caso de informes de Derechos Humanos en Chile.

(12) El 8 de octubre, un peloton fusiló a Jorge Vilugrón, atándolo a un poste de electricidad cerca del muelle. (Rettig 416)

(13) Detenido hacia mediados de diciembre de 1973 [...] dice haber sido torturada por un Capitán cuyo nombre no pudo saber [...]. (OEA)

(14) A esta comisión la asiste la convicción que Victor Hugo Careno fue ejecutado por agentes del estado que violaron su derecho a la vida. (Rettig 390)

Un grado menor de agentividad se expresa en las construcciones pasivas con un verbo intencional, pero sin agente $(15,16)$. En estos casos, si bien el verbo implica claramente la idea de un fusilamiento o de una ejecución - acciones intencionales - la ausencia del agente humano actor de la actividad implica una conceptualización en la cual el impacto de una persona humana en otra persona humana queda fuera del foco de atención explícito.

(15) Que Sócrates Ponce Pacheco, [...], fue fusilado el día 11 de septiembre [...] (OEA)

(16) El 12 de septiembre de 1973 fue ejecutado Benito TORRES TORRES, 57 años, instalador santiario. (Rettig 129)

Además de estas construcciones con verbos intencionales, aparecen en ambos informes construcciones con verbos que no implican necesariamente una intencionalidad ( $h a$ desaparecido en (17)). La intencionalidad solo se activa de modo indirecto por la conexión que se establece con un verbo intencional (detener en (17)) o un complemento de lugar tras la construcción pasiva (fue muerto en (18)). Incluso si el contexto histórico puede llevar a considerar que la desaparición y muerte fueron el resultado de un acto intencional, el texto del informe no presenta del modo claro al agente (aunque el espacio en (18) sí evoca uno, véase sección X y los análisis de agentes no prototípicos en Delbecque (2014)).

(17) Que Carlos Toranzos, (boliviano), fue detenido y ha desaparecido. (OEA)

(18) Una semana después, el 8 de noviembre de 1973, fue muerto, también en el Regimiento Arica de La Serena, José RODRIGUEZ ACOSTA [...]. (Rettig 276)

De forma semejante, se conceptualiza la violación del derecho a la integridad nombrando la actividad de abuso (apremios físicos y psicilógicos (19)) o la causa violenta de la muerte (por herida de bala tracto abdominal (20)). En estas oraciones el agente está encubierto y su presencia solo se infiere del conocimiento que poseemos: los apremios y las balas requieren de la intervención humana.

(19) Este derecho [derecho a la integridad personal] había sido y era directa y gravemente vulnerado por la práctica de apremios físicos y psicológicos de tratos crueles e inhumanos. (OEA) 
Barbara De Cock; Daniel Michaud Maturana. Marcela Alejandra Ruiz Zuñiga. Un enfoque cognitivo del análisis del discurso: el caso de informes de Derechos Humanos en Chile.

(20) El certificado de defunción acredita que murió "por herida de bala tracto abdominal". (Rettig 123)

Llama la atención que el Informe Rettig contiene estructuras en las cuales queda enteramente ausente la idea de intencionalidad y de violencia, como en (21) y (22). Podría entenderse que se trata de una muerte natural o de una desaparición voluntaria. Tales estructuras donde no se conceptualiza ningún agente, ni ninguna intencionalidad no se emplean en el informe de la OEA. Contrariamente a lo que se podría esperar, se observa, pues, que las estructuras de mayor encubrimiento son utilizadas en el Informe Rettig redactado al inicio de la transición y menos en el informe de la OEA redactado bajo la dictadura. Tales estrategias han sido descritas en otros contextos como eufemísticas (véase ALLAN; BURRIDGE, 1991; CRESPO, 2007; PIZARRO PEDRAZA; DE COCK, 2018). Este resultado sorprendente puede explicarse por el llamado 'Pacto del Silencio' durante la transición y un deseo de no nombrar a los perpetradores directamente. El informe de la OEA, en cambio, tuvo como función primordial la denuncia de la violencia, lo que puede explicar su preferencia por estructuras donde el carácter intencional de las violaciones a Derechos Humanos es parte de la función de denuncia de este informe.

(21) El 13 de septiembre de 1973 desaparece Eduardo Alejandro Alberto CAMPOS. (Rettig 132)

(22) El 16 de septiembre de 1973 murió Jorge Enrique DIAZ LOPEZ, 23 anos, transportista. (Rettig 143)

\subsection{Espacio}

La mención o no del espacio donde se ejerce la represión tiene importancia por varios motivos. El espacio puede evocar un marco y, de esta forma, sugerirnos un agente en particular. Sin embargo, observamos que la forma en que se mencionan los espacios bien puede facilitar la activación de un marco asociado con militares, policía u otros agentes del Estado, bien puede representar un lugar menos asociado con fuerzas del Estado. Ambos informes contienen numerosas referencias a lugares típicamente militares como regimiento, cuartel, Escuela de guerra, situando de esta forma la vulneración de Derechos Humanos en un contexto militar y sugiriendo, por ende, que los agentes son militares.

Ambos informes contienen también referencias a edificios no reconocidos como oficialmente militares, pero que son conceptualizados en el informe como lugares de detención y tortura, como por ejemplo la dirección Londres 38 en los ejemplos (23) y (24) (LAZARRA, 2011). Tal conceptualización de un edificio civil como lugar de tortura y/o 
Barbara De Cock; Daniel Michaud Maturana. Marcela Alejandra Ruiz Zuñiga. Un enfoque cognitivo del análisis del discurso: el caso de informes de Derechos Humanos en Chile.

detención importa en la denuncia de abusos de Derechos Humanos ya que el carácter civil de edificios fue utilizado por el régimen dictatorial para encubrir su responsabilidad en los abusos llevados a cabo en tales edificios por personal dirigido por las autoridades militares.

(23) La aplicación de apremios físicos y psicológicos en los siguientes establecimientos: Calle Londres 38 en Santiago; Escuela de Guerra de la FACh, en Santiago; una sección del Hospital Militar de Santiago; Departamento Central de Investigaciones en Santiago (local conocido como "La Patilla"); Tejas Verdes; y buque de la Armada "Esmeralda. (OEA)

(24) Los detenidos son traslados transitoriamente a una casa ubicada en calle Londres No 38 (lugar que posteriormente utilizó la DINA como centro de detención). (Rettig 192)

Por último, ambos informes mencionan ejemplos de abusos cometidos en la calle, un lugar que en principio no se identifica con las autoridades, pero que dado el Estado de Sitio y toque de queda invita a pensar que bien se podría tratar de actos cometidos por las autoridades, quienes controlaban la circulación de la ciudadanía en aquella época.

\section{Conclusiones}

En resumen, ambos documentos presentan las siguientes similitudes y diferencias. En ambos informes los perpetradores son representados como funcionarios (e.g. carabineros), y en ocasiones incluso como sujeto de la oración. También se describen las acciones de violencia, se identifican lugares de tortura (e.g. Escuela de Guerra, Regimientos) y se incluye a veces información sobre las detenciones. Las principales diferencias son que el informe de la OEA incluye además nombres concretos de perpetradores y/o describe físicamente al perpetrador, concretizando así su representación y aumentando la posibilidad de atribuirle responsabilidad. Además, en el informe de la OEA, no hay uso de expresiones verbales de muerte natural (e.g. muere, fallece) o desaparecer sin mayor información, lo que sí sucede en el Informe Rettig. También cabe destacar que el informe de la OEA representa los abusos de Derechos Humanos más explícitamente como actos intencionales violentos mientras el Informe Rettig en algunos casos presenta la muerte o desaparición como un fenómeno natural o voluntario. Sin embargo, incluso en esos casos hay que tomar en cuenta que el marco general del Informe Rettig es recopilar casos de abusos de Derechos Humanos y que, si bien algunas oraciones concretas no dejan claro el carácter violento de la muerte o desaparición, el marco textual general sí permite deducir el carácter violento. 
La comparación de las conceptualizaciones transmitidas en ambos informes permite establecer que realmente la especificidad del perpetrador es mayor en el informe OEA en el que las víctimas son una principal fuente de información. El análisis comparativo corrobora además que hay una evidente tendencia en el Informe Rettig a encubrir al perpetrador, por lo que se entiende el descontento de los familiares de las víctimas (DE COCK; MICHAUD, 2014). Si el valor informativo de los informes puede considerarse como una forma de contribuir al ejercicio del derecho a la memoria, el encubrimiento del perpetrador y la omisión de ciertas informaciones va precisamente en contra de ese derecho. Esas observaciones son una prueba más de la existencia del llamado Pacto del Silencio, es decir del pacto en la transición chilena para guardar silencio sobre la identidad de los responsables de los abusos de Derechos Humanos bajo la dictadura. Si bien son menos frecuentes que en el Informe Rettig, cabe destacar que también hay casos de encubrimiento del perpetrador en el informe de la OEA. Estos pueden deberse a falta de información sobre la identidad de los perpetradores o pueden ser considerados como estrategias de mitigación de responsabilidades en una época en la cual la dictadura negaba cualquier acusación.

A nivel metodológico y teórico, hemos mostrado mediante este análisis que el marco teórico de la lingüística cognitiva resulta provechoso para un análisis de discursos de gran relevancia social como estos informes. Efectivamente, el análisis del grado de especificidad, intencionalidad y agentividad con que se describe a los perpetradores permite constatar el empleo mayor de estrategias de encubrimiento de los perpetradores en el Informe Rettig. Los resultados obtenidos al respecto pueden también ser útiles para analizar otros tipos de discursos en las cuales la atribución o no atribución de la responsabilidad puede jugar un papel importante (véase PIZARRO PEDRAZA; DE COCK, 2018).

\section{Referencias}

ACCATINO, D., COLLINS, C. Truth, Evidence, Truth: The Deployment of Testimony, Archives and Technical Data in Domestic Human Rights Trials. Journal of Human Rights

Practice, v. 8, n.1, p. 81-100, 2016. Disponible en: <https://academic.oup.com/jhrp/article/8/1/81/1751614>. Consultado el: 19 marzo 2019. DOI: https://doi.org/10.1093/jhuman/huv019

ALLAN, K.; BURRIDGE, K. Euphemism \& dysphemism. Language used as shield and weapon. Oxford: Oxford University Press, 1991.

AMNESTY INTERNATIONAL. Chile: an Amnesty International Report. London: Amnesty International Publications, 1974. 
Barbara De Cock; Daniel Michaud Maturana. Marcela Alejandra Ruiz Zuñiga. Un enfoque cognitivo del análisis del discurso: el caso de informes de Derechos Humanos en Chile.

ANTHONISSEN, C.; BLOMMAERT, J. (Eds.). Critical linguistic perspectives on coping with traumatic pasts: case studies. Theme issue of Journal of Language and Politics, v. 5, n. 1, 2006. Disponible en: <https://benjamins.com/catalog/jlp.5.1>. Consultado el: 19 marzo 2019. DOI: https://doi.org/10.1075/jlp.5.1

BERNASCONI, O.; RUIZ, M.; LIRA, E. What defines the victims of human rights violations? The case of the Comité Pro Paz and Vicaría de la Solidaridad in Chile (19731992). In: DRULIOLLE, V.; BRETT, R. (Eds.). The Politics of Victimhood in Post-conflict Societies: Comparative and Analytical Perspectives. London: Palgrave MacMillan, 2018, p. 101-132. DOI: https://doi.org/10.1007/978-3-319-70202-5_5

BRUNNER, J. J. La cultura autoritaria en Chile. Santiago de Chile: FLACSO, 1981.

BRANTS, C.; KLEP, K. Transitional justice: history-telling, collective memory, and the victim-witness. International Journal of Conflict and Violence, v. 7, n.1, p. 36-49, 2013. Disponible en: <http://www.ijcv.org/index.php/ijcv/article/view/195>. Consultado el: 19 marzo 2019.

CABEZAS DEL FIERRO, C.; FIBLA CERDA, G.; JEREZ MUNDACA, C. Derecho a la memoria. ¿Derecho cultural implícito en el sistema constitucional chileno? Logos. Revista de lingüística, filosofía y literatura, v. 23, n. 2, p. 151-168, 2013. Disponible en: $<$ https://revistas.userena.cl/index.php/logos/article/view/358/414>. Consultado el: 19 marzo 2019.

COMISIÓN NACIONAL DE VERDAD Y RECONCILIACIÓN. Informe Rettig. Tomo 1, 1996. Disponibel en: <http://www.ddhh.gov.cl/ddhh_rettig.html>. Consultado el: 26 marzo 2004.

CRESPO FERNÁNDEZ, E. El eufemismo y el disfemismo. Procesos de manipulación y tabú en el lenguaje literario inglés. Alicante: Publicaciones Universidad de Alicante, 2007.

DE COCK, B.; MICHAUD MATURANA, D. La expresión de la agentividad en el Informe Rettig (Chile, 1991). Revista Internacional de Linguistica Iberoamericana, v. XII, n. 23, p. 123-140, 2014. Disponible en: <https://www.researchgate.net/publication/268277445_La_expresion_de_la_agentividad_en_ el_Informe_Rettig_Chile_1991>.Consultado el: 19 marzo 2019.

DE COCK, B.; MICHAUD MATURANA, D. Discursive construction of human rights violations: the case of the Chilean Rettig report. Text \& Talk: an interdisciplinary journal of language, discourse \& communication studies, v. 38, p. 1-21, 2018. Disponible en: $<$ https://www.degruyter.com/view/j/text.2018.38.issue-1/text-2017-0028/text-20170028.xml>. Consultado el: 19 marzo 2019. DOI: https://doi.org/10.1515/text-2017-0028

DELBECQUE, N. Passive Voice and Causal Roles in Spanish. In: DELBECQUE, N.; LAHOUSSE, K.; VAN LANGENDONCK, W. (Eds.). Nuclear and non-nuclear cases. Amsterdam: J. Benjamins, 2014, p. 217-318. DOI: https://doi.org/10.1075/cagral.6.07del

DORAT GUERRA, C.; WEIBEL BARAHONA, M. Asociación ilícita. Los archivos secretos de la dictadura. Santiago, Chile: Ceibo Ediciones, 2012. 
Barbara De Cock; Daniel Michaud Maturana. Marcela Alejandra Ruiz Zuñiga. Un enfoque cognitivo del análisis del discurso: el caso de informes de Derechos Humanos en Chile.

FERRARA, A. Evaluando los efectos de las diferentes Comisiones de la Verdad: el caso chileno. In: JIMENO ARANGUREN, R. (Ed.), Justicia transicional: historia y actualidad. Navarra: Thomson Reuters Aranzadi, 2017, p. 213-239.

FILLMORE, Charles J. Form and meaning in language. v. 1. Papers on Semantic Roles. Stanford: CSLI Publications, 2003.

GRANDIN, G. The Instruction of Great Catastrophe: Truth Commissions, National History, and State Formation in Argentina, Chile, and Guatemala. American Historical Review, p.46-67, February 2005. Disponible en: <https://academic.oup.com/ahr/article/110/1/46/12680>. Consultado el: 19 marzo 2019. DOI: https://doi.org/10.1086/531121

GROPPO, B. Dictaduras miltares, archivos de movimientos políticos y sociales y archivos de la represión en América Latina. In: ACUÑA, M. G.; FLIER, P. (Eds.). Archivos y memoria de la represión en América Latina (1973-1990). Santiago: Lom Ediciones, 2016, p. 31-53.

GUTHREY, H. L. Victim healing and truth commissions: transforming pain through voice in Solomon Islands and Timor-Leste. v. 11. Cham: Springer, 2015. DOI: https://doi.org/10.1007/978-3-319-12487-2

HART, C.,; LUKEŠ, D. Cognitive Linguistics in Critical Discourse Analysis: Application and Theory. Newcastle: Cambridge Scholars Publishing, 2007.

HAU, B. La defensa de los Derechos Humanos del Departamento Jurídico del Comité Pro Paz y de la Vicaría de la Solidaridad. Santiago: Tesis Universidad Alberto Hurtado. 2005. Disponible en: <http://tesis.museodelamemoria.cl/Tesis_PDF/Tesis\%20Hau.pdf>. Consultado el: 23 abril 2019.

HAYNER, P. Verdades Innombrables. El Reto de las comisiones de la verdad. México: Fondo de la Cultura Económica, 2008.

JOHANSSON MÁRQUEZ, M. T. Discurso e ideología en la problemática de las violaciones a los Derechos Humanos: un análisis crítico de editoriales sobre el informe Rettig. Unpublished Magister thesis Universidad de Chile, 2001.

KNAPTON, O.; RUNDBLAD, G. Metaphor, discourse dynamics and register: applications to written descriptions of mental health problems. Text \& Talk, v. 38, n. 3, p. 389-410, 2018. Disponible en: <https://www.degruyter.com/view/j/text.ahead-of-print/text-2018-0005/text2018-0005.xml>. Consultado el: 26 marzo 2019. DOI: https://doi.org/10.1515/text-2018-0005

LAZARRA, Michael J. Dos propuestas de conmemoración pública: Londres 38 y el Museo de la Memoria y los Derechos Humanos (Santiago de Chile). A Contracorriente. Una revista de historia social $\mathbf{y}$ literatura en América Latina, v. 8, n. 3, p. 55-90, 2011. Disponible en: <https://projects.ncsu.edu/project/acontracorriente/spring_11/articles/Lazzara.pdf >. Consultado el: 26 marzo 2019. 
Barbara De Cock; Daniel Michaud Maturana. Marcela Alejandra Ruiz Zuñiga. Un enfoque cognitivo del análisis del discurso: el caso de informes de Derechos Humanos en Chile.

LOVEMAN, B., LIRA, E. Las ardientes cenizas del olvido: vía chilena de reconciliación política 1932-1994. Santiago: Lom Ediciones, 2000.

LOVEMAN, B., LIRA, E. Políticas de la Verdad en Chile: 1891-1991. In: LIRA, E.; LOVEMAN, B.; MIFSUD, T.; SALVAT, P. (Eds.), Historia, política y ética de la verdad en Chile, 1891-2001: reflexiones sobre la paz social y la impunidad. Santiago: Lom Ediciones, 2001, p. 19-110.

MACDERMOT, N.; MADLENER, K.; OLIVER, C. Final Report of Mission to Chile to study the Legal System and the Protection of Human Rights. Geneve: International Commission of Jurists, 1974.

OEA. Comisión Interamericana de Derechos Humanos. Informe OEA. Informe sobre la situación de los Derechos Humanos en Chile. 1974. Disponible en: <http://www.cidh.org/countryrep/Chile74sp/Indice.htm>. Consultado el: 26 marzo 2019.

OTEÍZA, T. Evaluative patterns in the official discourse of human rights in Chile: giving value to the past and building historical memories in society. DELTA [online], n. 25, p. 609640, 2009. Disponible en: <http://www.scielo.br/scielo.php?script=sci_arttext\&pid=S010244502009000300004>. Consultado el: 26 marzo 2019. ${ }^{4}$ DOI: https://doi.org/10.1590/S010244502009000300004

OTEÍZA, T. Patrones valorativos en el discurso oficial de Derechos Humanos en Chile: dando valor al pasado y construyendo memorias históricas en la sociedad. Discurso y Sociedad, v. 4, n. $1, \quad$ p. 151-183, 2010. Disponible en: <http://www.scielo.br/scielo.php?script=sci_abstract\&pid=S0102-

$44502009000300004 \& \operatorname{lng}=\mathrm{en} \& n r m=i s o \& t \operatorname{lng}=\mathrm{es}>$. Consultado el: 26 marzo 2019.

PIZARROPEDRAZA, A.; DE COCK, B. Taboo effects at the syntactic level: reducing agentivity as a euphemistic strategy. Pragmatics: quarterly publication of the International Pragmatics Association, v. 28, n. 1, p. 113-138, 2018. Disponible en: <https://benjamins.com/catalog/prag.17001.piz/fulltext/prag.17001.piz.pdf>. Consultado el: 26 marzo 2019. DOI: https://doi.org/10.1075/prag.17001.piz

RUIZ, M.; BERNASCONI, O. Reports on Categorization and classification of Human Rights violations in Chile, 1974 - 1978. Discourse \& Society, v. 30, n. 1, p. 44-63, 2019. Disponible en: <https://journals.sagepub.com/doi/abs/10.1177/0957926518801081>. Consultado el: 26 marzo 2019. DOI: https://doi.org/10.1177/0957926518801081

SAMBRE, P. The Multimodal Representation of Italian Anti-Mafia Discourse: Foregrounding Civil Resistance and Interlocution in Two Global English Video Reportages. I-LanD Journal: Identity, Language and Diversity, p. 38-59, 2017. Disponible en: $<$ https://limo.libis.be/primoexplore/fulldisplay?docid=LIRIAS1668228\&context=L\&vid=Lirias\&search_scope=Lirias\&t $\mathrm{ab}=$ default_tab\&lang=en_US\&fromSitemap=1 >. Consultado el: 26 marzo 2019.

SAMBRE, P.; FEYAERTS, K. Embodied musical meaning-making and multimodal viewpoints in a trumpet master class. Journal of Pragmatics, n. 122, p. 10-23, 2017.

\footnotetext{
${ }^{4}$ Una traducción al español está disponible en:

<http://www.dissoc.org/ediciones/v04n01/DS4\%281\%290teiza.pdf>.
} 
Disponible

en:

<https://www.sciencedirect.com/science/article/abs/pii/S037821661630385X>. Consultado el: 26 marzo 2019. DOI: https://doi.org/10.1016/j.pragma.2017.09.004

TALMY, L. Toward a cognitive semantics. v. 1. Cambridge MA: MIT Press, 2000. DOI: https://doi.org/10.7551/mitpress/6847.001.0001

VALDIVIA ORTIZ DE ZÁRATE, V. Terrorism and Political Violence during the Pinochet Years: Chile, 1973-1989. Radical History Review, v. 85, n. 1, p. 182-190, 2003. Disponible en:

<http://www.umass.edu/legal/Benavides/Fall2004/397U/Electronic\%20Reserve\%20Legal\%2 0397U/13\%200rtiz\%20Chile.pdf>. Consultado el: 26 marzo 2019. DOI: https://doi.org/10.1215/01636545-2003-85-182

VALDIVIA ORTIZ DE ZÁRATE, V. “Estamos en guerra, señores!”. El régimen militar de Pinochet y el "pueblo", 1973-1980. Historia, v. I, n. 43, p. 163-201, 2010. Disponible en: <http://www.redalyc.org/articulo.oa?id=33416405005>. Consultado el: 26 marzo 2019.

WILSON, R. The politics of truth and reconciliation in South Africa: Legitimizing the post-apartheid state. Cambridge University Press, 2001. DOI: https://doi.org/10.1017/CBO9780511522291

WINN, P. El pasado está presente. Historia y memoria en el Chile contemporáneo, 2007. Disponible en: $<$ http://www.historizarelpasadovivo.cl/es_resultado_textos.php?categoria=Chile\%3A+los+ca minos+de+la+historia+y+la+memoria\&titulo $=\mathrm{El}+$ pasado+est $\% \mathrm{E} 1+$ presente. + Historia $+\mathrm{y}+\mathrm{me}$ moria+en+el+Chile+contempor\%E1neo>. Consultado el: 26 marzo 2019.

Recebido em: 27 de março de 2019

Aceito em: 2 de maio de 2019 